\title{
Análisis de la actividad matemática mediante dos herramientas teóricas: Registros de representación semiótica y configuración ontosemiótica
}

\author{
Juan D. Godino, Universidad de Granada (España) \\ Miguel R. Wilhelmi, Universidad Pública de Navarra (España) \\ Teresa F. Blanco, Universidad de Santiago de Compostela (España) \\ Ángel Contreras, Universidad de Jaén (España) \\ Belén Giacomone, Universidad de Granada (España)
}

Recibido el 30 de abril de 2016; aceptado el 12 de julio de 2016

\begin{abstract}
Análisis de la actividad matemática mediante dos herramientas teóricas: Registros de representación semiótica y configuración ontosemiótica

\section{Resumen}

Para comprender las dificultades y conflictos de aprendizaje, es necesario analizar las tareas matemáticas y los diversos modos de abordarlas por los estudiantes. Dicho análisis, que precisa herramientas teóricas específicas para su realización, aporta información útil para el propio diseño de las tareas y la gestión de los conocimientos en el aula. En este trabajo realizamos el análisis de una tarea que requiere la formulación de una conjetura y su demostración haciendo uso de representaciones figurales y algebraicas, aplicando dos herramientas teóricas diferentes: las nociones de registro de representación semiótica y de configuración ontosemiótica. Los resultados revelan algunas complementariedades que nos permitieron mostrar la potencial utilidad de los análisis epistémico y cognitivo realizados. Se trata de mostrar la potencial sinergia existente entre dichas herramientas y la posibilidad de avanzar en la articulación de los marcos teóricos correspondientes.
\end{abstract}

Palabras claves. Prácticas matemáticas; registros de representación; configuración ontosemiótica; análisis cognitivo y epistémico; articulación de teorías.

Análise de atividade matemática através de duas ferramentas teóricas: Registos de representação semiótica e configuração ontossemiótica

\section{Resumo}

Para compreender as dificuldades e conflitos de aprendizagem, é necessário analisar as tarefas matemáticas e os diversos modos de resolução por parte dos estudantes. Esta análise, que precisa de ferramentas teóricas específicas para a sua realização, fornece informação útil para o próprio desenho de tarefas e para a gestão de conhecimentos em sala de aula. Neste trabalho realizamos a análise de uma tarefa que requer a formulação de uma conjetura e sua demonstração, fazendo uso de representações figurais e algébricas, aplicando duas ferramentas teóricas diferentes: as noções de registo de representação semiótica e de configuração ontossemiótica. Os resultados revelam algumas complementaridades que nos permitem manifestar o potencial da ontosemiótica. Avances de Investigación en Educación Matemática, 10, 91-110 
utilização da análise epistémica e cognitiva realizada. Isto mostra o potencial da sinergia existente entre as ferramentas teóricas referidas e a possibilidade de avançar na articulação dos marcos teóricos correspondentes.

Palavras chave. Práticas matemáticas; registos de representação; configuração ontosemiótica; análise cognitiva e epistémica; articulação de teorias.

Analysing mathematical activity through two theoretical tools: registers of semiotic representation and onto-semiotic configuration

\section{Abstract}

To understand the difficulties and conflicts of learning is necessary to analyse the mathematical tasks and the various ways of addressing them by students. This analysis provides information about the design of the tasks and the management of knowledge in the classroom, being necessary to apply specific theoretical tools for its realisation. In this paper, we analyse a task that requires the formulation of a conjecture and its proof using figural and algebraic representations, and applying two different theoretical tools: the notions of semiotic representation register and onto-semiotic configuration. The results reveal some complementarities that allowed us to show the potential utility of the epistemic and cognitive analysis carried out. The aim is to show the potential synergy between these tools and the possibility to progress in the articulation of the corresponding theoretical frameworks.

Key words. Mathematical practices; representation registers; onto-semiotic configuration; cognitive and epistemic analysis; networking theories.

Analyse de l'activité mathématique par le biais de deux outils théoriques: les registres de représentation sémiotique et la configuration ontologique et sémiotique

\section{Résumé}

Pour comprendre les difficultés et les conflits d'apprentissage, il faut analyser les tâches mathématiques et les différentes façons que les étudiants utilisent pour les résoudre. Cette analyse, qui a besoin des outils théoriques spécifiques pour sa mise en ouvre, fournit des informations utiles pour la conception des tâches et pour la gestion des connaissances dans le système didactique. Dans cet article, on analyse la formulation d'une conjecture pour la réalisation d'une tâche, ainsi que sa démonstration par le biais de représentations figurales et algébriques. Pour atteindre cet objectif, on utilise deux notions théoriques différentes: les registres de représentation sémiotique et la configuration ontologique et sémiotique. Les résultats révèlent des complémentarités entre les outils théoriques utilisés et leur intérêt pour des analyses épistémiques et cognitives complexes. On a donc montré la synergie potentielle entre ces outils et on a aussi trouvé des solutions qui visent l'articulation des cadres théoriques.

Paroles clés. Pratiques mathématiques; registres de représentation; configuration ontologique et sémiotique; analyse cognitive et épistémologique; articulation des théories.

\section{Introducción}

La teoría de los Registros de Representación Semiótica (TRRS) (Duval, 1995; 2006) incluye nociones que permiten el análisis de los diversos tipos de representaciones materiales usadas en la realización de tareas matemáticas, las transformaciones de las mismas y el papel que juegan en la comprensión de las matemáticas. La disponibilidad y uso de diversos sistemas de representación semiótica, sus transformaciones y conversiones, se consideran imprescindibles para la comprensión, construcción y comunicación de las matemáticas. Asimismo, se asume que la producción y aprehensión de representaciones materiales no es espontánea y su dominio debe ser previsto en la enseñanza.

Por su parte, el Enfoque Ontosemiótico del conocimiento y de la instrucción matemáticos (EOS) (Godino, Batanero y Font, 2007) ha puesto el énfasis en los diversos tipos de objetos matemáticos, su naturaleza y su emergencia de las prácticas matemáticas. El lenguaje, en sus 
diversas modalidades, se incluye como un tipo de objeto primario y se reconoce su papel (representacional e instrumental) como faceta ostensiva de los objetos matemáticos. En este marco, para analizar la actividad matemática, se definen unos objetos primarios (lenguajes, situaciones, conceptos, proposiciones, procedimientos y argumentos), siendo los lenguajes un aspecto central del aprendizaje. De hecho, el EOS propone un conjunto de herramientas que permite el análisis contextual de los objetos, determinando los procesos donde son movilizados o emergen. Además se ponen en relación unos procesos con otros, permitiendo un análisis de las condiciones de comunicación y comprensión de las matemáticas.

El objetivo de este trabajo es estudiar las posibilidades de articulación de estos dos marcos teóricos, siguiendo los pasos marcados por los recientes estudios sobre comprensión, comparación y articulación de teorías (Bikner-Ahsbahs \& Prediger, 2014; Prediger, BiknerAhsbahs, \& Arzarello, 2008), y teniendo en cuenta los resultados de la investigación iniciada por Pino-Fan, Guzmán, Duval y Font (2015) sobre la articulación de la TRRS y el EOS. En una primera aproximación se puede prever que la noción de registro de representación semiótica, sus diversos tipos y las operaciones de tratamiento y conversión entre registros permite desarrollar el análisis de los elementos lingüísticos, siendo por tanto un enriquecimiento del EOS. Paralelamente, la noción de configuración de objetos y procesos puede aportar un enriquecimiento de la TRRS, al permitir un análisis detallado de los conocimientos implicados en las transformaciones entre registros de representación que se realizan en las prácticas matemáticas (Pino-Fan et al., 2015).

Aunque en los supuestos ontológicos y semióticos de ambos marcos teóricos se encuentran diferencias importantes se parte de la hipótesis de que es posible una cierta articulación de los mismos, lo que permitiría hacer análisis cognitivos y epistémicos más detallados de la actividad matemática, $\mathrm{y}$, en consecuencia, contribuir a la comprensión de los procesos de enseñanza y aprendizaje.

En la siguiente sección hacemos una síntesis de los supuestos y herramientas de la TRRS y en la siguiente hacemos lo mismo con los correspondientes al EOS. En la Sección 4 aplicamos conjuntamente estas herramientas para analizar una tarea matemática, lo cual ayuda a identificar algunas concordancias y complementariedades. Se prefiere, en primer lugar, realizar el análisis sobre una resolución epistémica de la tarea, con el fin de explicitar de manera completa las prácticas institucionales requeridas y procurando, además, aplicar de manera sistemática la noción de función semiótica, tanto en su interpretación referencial (semántica) como operacional (pragmática). Seguidamente se realiza el análisis cognitivo de la respuesta de un estudiante de máster a la tarea, aplicando las mismas herramientas teóricas y mostrando las posibilidades ofrecidas por el análisis epistémico previo para interpretar y comprender la solución dada por el estudiante. Por último, en las secciones 6 y 7, se profundiza en la comparación y articulación de los dos marcos teóricos.

\section{Supuestos y herramientas de la TRRS}

En la perspectiva semiótica - cognitiva adoptada por la TRRS se plantea abordar los problemas de aprendizaje de las matemáticas a partir de los distintos tipos de signos que se usan en la práctica matemática (atendiendo a su función y naturaleza). Tales signos son entendidos como representaciones materiales o externas, más que como representaciones mentales, considerándose que el modo de acceso a los objetos matemáticos, a diferencia de los objetos de otros campos de conocimiento científico, nunca puede ser directo mediante la percepción, sino haciendo uso necesariamente de las representaciones de tales objetos. Así, en la TRRS son claves las nociones de semiosis y noesis. 
Se llama semiosis a la aprehensión o la producción de una representación semiótica, y noesis a los actos cognitivos como la aprehensión conceptual de un objeto, la discriminación de una diferencia o la comprensión de una inferencia (Duval, 1995, p. 3).

Contrariamente a muchos trabajos psicológicos y didácticos que consideran la noesis como independiente de la semiosis o, que al menos, la dirige, R. Duval considera que la noesis está íntimamente ligada a la movilización y a la articulación cuasi-inmediata de varios registros de representación semiótica. Se atribuye un papel esencial no solo al uso de diferentes sistemas de representación semiótica (SRS) para el trabajo matemático, sino al tratamiento de los signos dentro de cada sistema y la conversión entre diferentes SRS.

¡El papel que los signos juegan en matemáticas no es ser sustituidos por otros objetos sino por otros signos! Lo que importa no es la representación sino sus transformaciones. Contrariamente a otras áreas del conocimiento científico, los signos y la representación semiótica de las transformaciones son el corazón de la actividad matemática (Duval, 2006, p. 107).

Para que un conjunto estructurado de signos sea considerado un Registro de Representación Semiótica (RRS) R. Duval requiere que en su seno se puedan realizar tres actividades cognitivas fundamentales:

En primer lugar, constituir una traza o un conjunto de trazas perceptibles que sean identificables como una representación de cualquier cosa en un sistema determinado. A continuación, transformar las representaciones mediante las únicas reglas propias del sistema de manera que se obtengan otras representaciones que pueden constituir un aporte de conocimiento con relación a las representaciones iniciales. Finalmente, convertir las representaciones producidas en un sistema en representaciones de otro sistema, de tal manera que estas últimas permitan explicitar otras significaciones relativas a lo que se representa (Duval, 1995, p. 21).

Como ejemplos de tales RRS se tienen, la lengua natural (oral, escrita); representaciones numéricas (entera, fraccionaria, decimal); representaciones figurales o gráficas (lineales, planas o espaciales) y representaciones alfanuméricas (algebraicas). Se reconoce la posición dominante del RRS de la lengua natural, en tanto metalenguaje de todos los lenguajes y de él mismo, es decir, como filtro de toda nuestra experiencia con el mundo natural, social y simbólico o cultural.

Las transformaciones posibles entre representaciones pueden ser de dos tipos:

- Tratamiento. La actividad supone una transformación entre representaciones de un mismo RRS.

- Conversión. La actividad supone una transformación entre representaciones de distintos RRS, siendo, en este caso, esencial la articulación de los registros.

En el enunciado y solución de una tarea matemática pueden intervenir distintas representaciones semióticas pertenecientes a diferentes RRS, las cuales pueden sufrir distintos tratamientos o conversiones. Dos representaciones se dice que son congruentes cuando cumplen tres condiciones: correspondencia semántica entre las unidades significantes que las constituyen, mismo orden posible de aprehensión de estas unidades en las dos representaciones, y para convertir una unidad significante de la representación de partida se tiene una única unidad significante en la representación de llegada (Duval, 1995, p. 6). Pero cuando uno de estos tres criterios no se verifica, las representaciones dejan de ser congruentes entre sí, y el paso de una a la otra no tiene nada de inmediato. 
El análisis semiótico - cognitivo de la actividad matemática llevado a cabo dentro de la TRRS se centra en la identificación de los distintos registros usados y sus transformaciones, según las reglas propias de cada RRS. Duval (2006) concibe el objeto matemático como "el invariante de un conjunto de fenómenos o el invariante de alguna multiplicidad de posibles representaciones" (p. 129), e insiste en no confundir el objeto matemático con sus diversas representaciones. Esto le lleva a plantear la paradoja cognitiva del aprendizaje matemático:

El problema crucial de la comprensión matemática para los estudiantes, en cualquier nivel del currículo, surge del conflicto cognitivo entre estos dos requerimientos opuestos: cómo pueden distinguir el objeto representado de la representación semiótica usada si no pueden tener acceso al objeto matemático sino por medio de las representaciones semióticas (Duval, 2006, p. 107).

Un sujeto tendrá acceso a un objeto representado solamente si se cumplen dos condiciones: 1) que disponga de al menos dos registros diferentes para representar el objeto; y 2) que pueda pasar de manera natural de un registro a otros, aun sin ser consciente de las representaciones que está articulando. Si esto no ocurre, la representación y el objeto representado (notación y contenido) se confunden.

La actividad conceptual no puede ser aislada de la actividad semiótica porque la comprensión conceptual aparece ligada al descubrimiento de una invariancia entre representaciones semióticas heterogéneas (Duval, 1995, p. 1).

Los planteamientos expuestos han posibilitado la constitución de una herramienta didáctica (RRS) que pone de manifiesto la necesidad de integrar la manipulación de registros (ostensivos) y la interpretación de esta manipulación (significado). Así, se observan dos niveles de análisis: uno, referido a los "objetos" (ostensivos - materiales o conceptuales) matemáticos involucrados; otro, sobre las relaciones entre ellos y su función contextual en la actividad matemática, discriminando, en particular, los objetos emergentes. Estos dos niveles deben ser integrados, para lo cual es preciso determinar las configuraciones de objetos y la función significante que cumple cada uno de ellos (Montiel, Wilhelmi, Vidakovic, \& Elstak, 2009).

Por otro lado, el postulado de la TRRS sobre la necesidad para el acceso a un objeto de que el sujeto disponga de al menos dos registros diferentes para representarlo no es general. Por ejemplo, cuando un niño en el aula de tres años de Educación Infantil recibe un conjunto de triángulos, cuadrados y círculos de diferentes tamaños, colores y grosores y, dado un ejemplar cualquiera de cada figura, se le solicita "coge todos los que tienen la misma forma", el niño realiza una clasificación del conjunto, que es una abstracción de las propiedades de las figuras. Aquí el niño no dispone de otro registro de representación de las figuras, que no sea el material-manipulable (como representante de la clase "cuadrado", "triángulo" y "círculo"). De hecho, el niño puede no saber nombrar las figuras en lenguaje natural y, en todo caso, lo figural está condicionado por la psicomotricidad fina (Dickson, Brown, \& Gibson, 1991). Sin embargo, es capaz de apresar características suficientes de los objetos, abstrayendo otras características físicas, para realizar la tarea de clasificación. Es pues necesario para describir este aprendizaje un análisis que excede el cambio de registros.

\section{Supuestos y herramientas del EOS: la noción de configuración ontosemiótica}

Godino (2012) presenta el EOS como un enfoque teórico inclusivo para la investigación en didáctica de las matemáticas. El EOS aporta herramientas para abordar cuestiones de tipo epistemológico, cognitivo e instruccional en los procesos de enseñanza y aprendizaje de las matemáticas, desde una aproximación antropológica y semiótica. En la Figura 1 se destacan 
como elementos claves de la modelización epistemológica y cognitiva del conocimiento matemático las nociones de práctica, objeto, proceso (secuencia de prácticas de las que emerge el objeto) y función semiótica.

Interpretando la noción de "función de signo" de Hjemslev (1943) y "función semiótica" de Eco (1976), en el EOS se entiende una función semiótica como la correspondencia o relación de dependencia entre una entidad antecedente (expresión, significante) y otra consecuente (contenido, significado) establecida por un sujeto (persona o institución) según una determinada regla, hábito o criterio establecida en un acto de interacción comunicativa. Así mismo, de acuerdo con la semiótica de Peirce (1978), se asume que tanto la expresión (antecedente de una función semiótica) y el contenido (consecuente) pueden ser cualquier tipo de entidad de las introducidas en el EOS: un sistema de prácticas o cualquiera de las entidades primarias que intervienen en las prácticas (problemas, lenguajes, conceptos, proposiciones, procedimientos, argumentos). Como tales entidades se pueden contemplar desde diferentes puntos de vista duales (personal- institucional, unitario- sistémico, ostensivo - no ostensivo, particular-general), los significados también pueden ser considerados desde dichos puntos de vista. El significado de un objeto (una palabra, un concepto, etc.), entendido como el uso o rol que dicho objeto desempeña en un contexto o juego de lenguaje específico, queda contemplado como el caso en el que el contenido de la función semiótica es un sistema de prácticas (significado pragmático $\mathrm{u}$ operacional). Se tiene de esta manera una conceptualización muy general y operativa de nociones claves en psicología y educación, como son las de significado y representación.

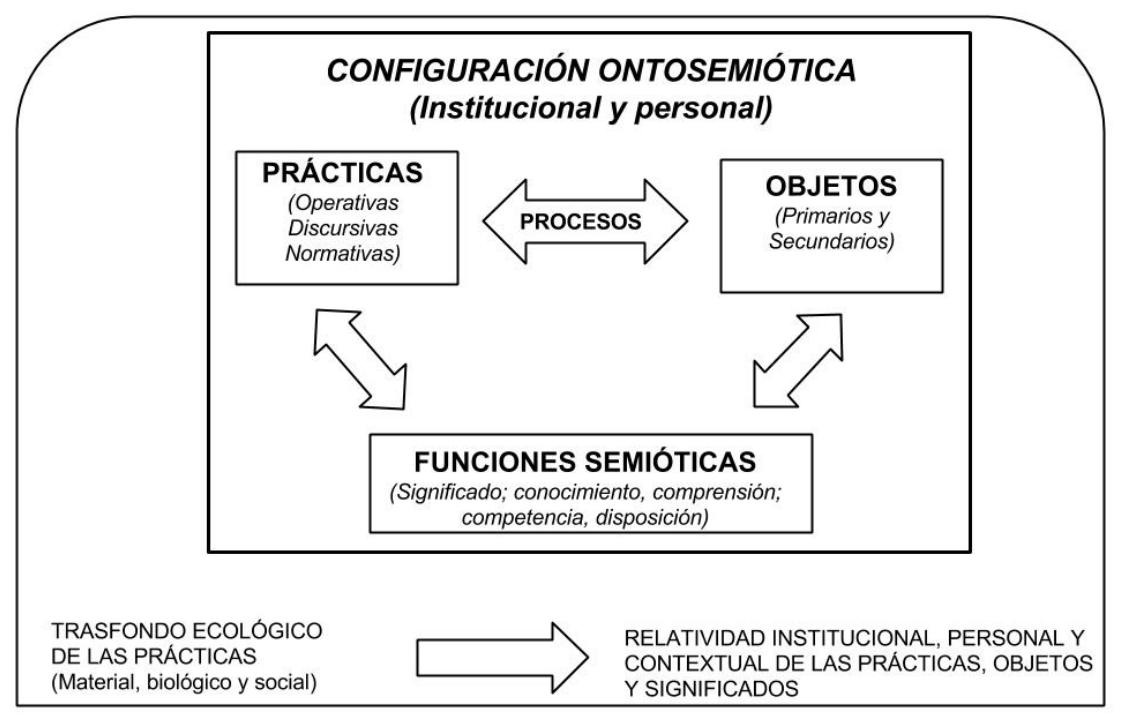

Figura 1. Entidades primarias de la ontología y epistemología EOS.

El EOS incluye una tipología explícita de objetos (y de sus respectivos procesos), que condiciona la descripción y el análisis de la actividad matemática; estos son:

- Lenguajes (términos, expresiones, notaciones, gráficos) en sus diversos registros (escrito, oral, gestual, etc.).

- Situaciones-problemas (aplicaciones intra o extra-matemáticas, ejercicios).

- Conceptos- definición (introducidos mediante definiciones o descripciones) (recta, punto, número, media, función).

- Proposiciones (enunciados sobre conceptos). 
- Procedimientos (algoritmos, operaciones, técnicas de cálculo).

- Argumentos (enunciados usados para justificar o explicar las proposiciones y procedimientos, deductivos o de otro tipo).

Estos objetos pueden ser analizados desde cinco pares de puntos de vista duales (Figura 2) (Font, Godino, \& Gallardo, 2013), según su función contextual y funcional en las prácticas matemáticas, así como de los procesos matemáticos relacionados.

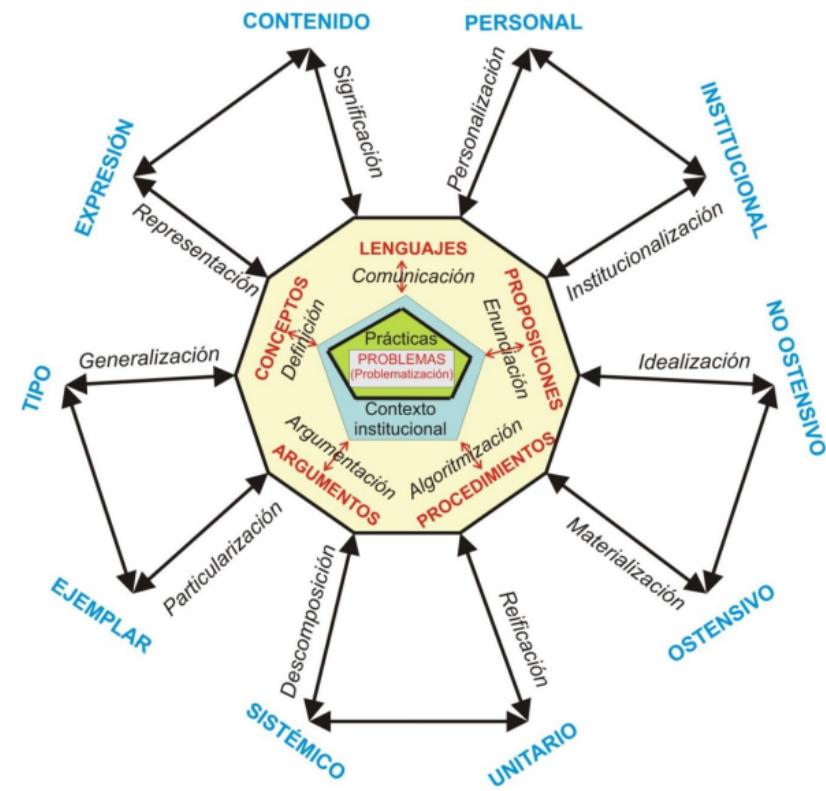

Figura 2. Objetos y procesos que intervienen en las prácticas matemáticas

Tanto las dualidades como los objetos se pueden analizar desde la perspectiva procesoproducto. La emergencia de los objetos primarios (problemas, definiciones, proposiciones, procedimientos y argumentos) tiene lugar mediante los respectivos procesos matemáticos de comunicación, problematización, definición, enunciación, elaboración de procedimientos (algoritmización, rutinización, ...) y argumentación. Por otra parte, las dualidades dan lugar a los siguientes procesos cognitivos/epistémicos: institucionalización - personalización; generalización - particularización; análisis/descomposición - síntesis/reificación; materialización /concreción - idealización/ abstracción; expresión/representación significación.

La noción de sistema semiótico de la TRSS se interpreta en el EOS en términos de las configuraciones de objetos y procesos, que no quedan restringidas a conjuntos de signos relacionados. Así, estos sistemas están formados por la configuración de objetos que intervienen y emergen en los sistemas de prácticas, junto con los procesos de interpretación que se establecen entre los mismos (esto es, incluyendo la red de funciones semióticas que relacionan los objetos constituyentes de la configuración) (Godino, Font, Wilhelmi, \& Lurduy, 2011).

Las configuraciones epistémicas /cognitivas están constituidas por los elementos lingüísticos de distinta naturaleza (gestos, palabras, inscripciones, ...) y también por los objetos conceptuales, proposiciones, procedimientos y argumentos, junto con los problemas que constituyen la razón de ser de la actividad, y las relaciones que se establecen entre los 
constituyentes mencionados. Son por lo tanto heterogéneas, multimodales y dinámicas, ya que cambian y se enriquecen según evolucionan los procesos instruccionales.

\section{Análisis conjunto de una tarea mediante la TRRS y el EOS}

En este apartado vamos a analizar el enunciado y una posible solución (experta o ideal) de una tarea aplicando herramientas teóricas de la TRRS y del EOS. Se aportará un análisis a partir de las herramientas de la TRRS, identificando ciertas cuestiones abiertas que serán abordadas con las herramientas de EOS. Este ejemplo integra distintos tipos de lenguajes (natural, figural, numérico y simbólico-literal), resaltando la importancia de la visualización en la adquisición de conocimientos matemáticos siempre que estas sean internas y globales, detectándose conflictos en la actividad matemática si son externas y parciales (Ortega \& Pecharromán, 2015).

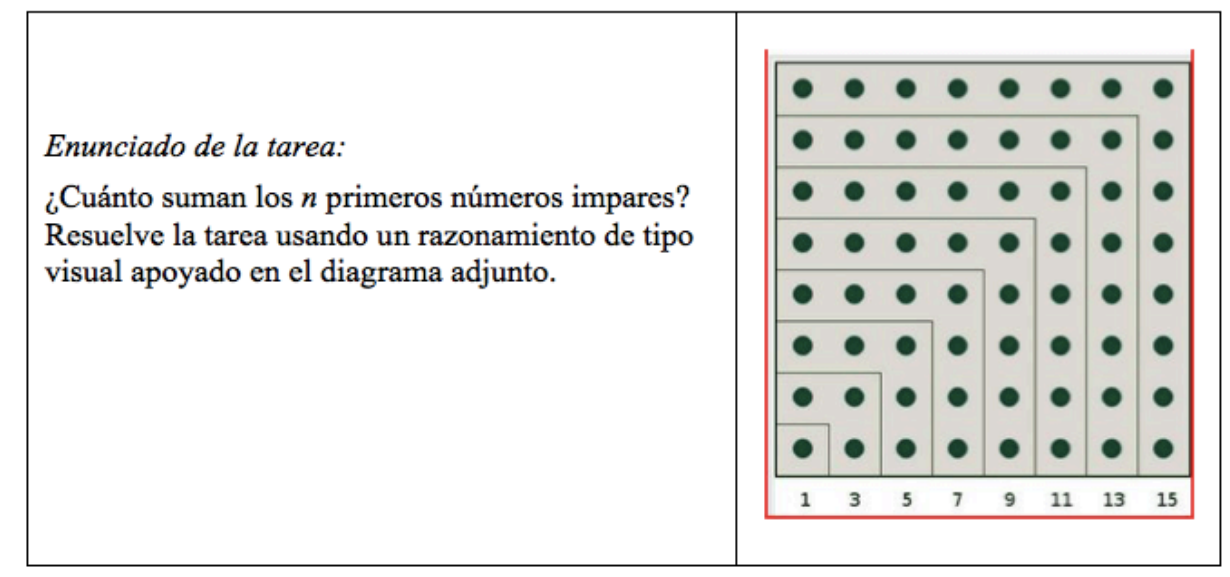

Figura 3. Enunciado de una tarea visual - algebraica.

\subsection{Sistema de acciones que componen la solución}

La representación de la Figura 3 presenta una "demostración visual" clásica de este teorema. Se muestra visualmente la suma de los ocho primeros números impares, de la cual se puede formular una conjetura para el caso de la suma de los primeros $n$ números impares: $1+3+\cdots+(2 n-1)=n^{2}$. La demostración de esta fórmula para cualquier número natural puede obtenerse aplicando el principio de inducción matemática. Para que el diagrama de la Figura 3 desempeñe su función explicativa se considera necesario realizar una secuencia de prácticas o acciones similares a las que indicamos a continuación:

1. El diagrama de la Figura 3 se debe descomponer en 8 sub-diagramas (Figura 4) según las líneas angulares interiores. La secuencia de las ocho figuras establece una correspondencia entre los primeros ocho números impares y el número de orden de la figura.

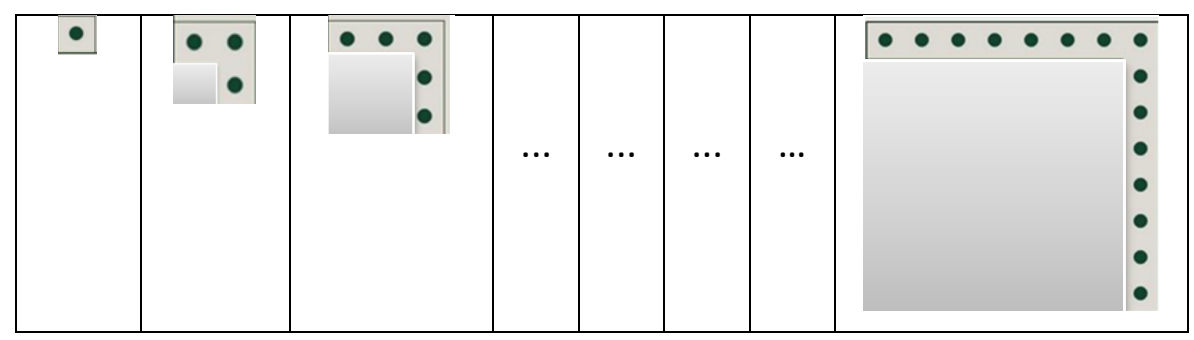




\begin{tabular}{|c|c|c|c|c|c|c|c|}
\hline 1 & 3 & 5 & 7 & 9 & 11 & 13 & 15 \\
\hline 1 & 2 & 3 & 4 & 5 & 6 & 7 & 8 \\
\hline
\end{tabular}

Figura 4. Distinción de 8 casos

2. En cada caso se calcula el número de puntos de dos formas: como área discreta de un cuadrado (números cuadrados) y como suma de números impares consecutivos (Figura 5).

\begin{tabular}{|c|c|c|c|c|c|c|c|}
\hline$\bullet$ & \begin{tabular}{ll|}
$\bullet$ & $\bullet$ \\
$\bullet$ & $\bullet$ \\
\end{tabular} & $\begin{array}{|lll|}\bullet & \bullet & \bullet \\
\bullet & \bullet & \bullet \\
\bullet & \bullet & \bullet \\
\end{array}$ & \begin{tabular}{llll|}
$\bullet$ & $\bullet$ & $\bullet$ & $\bullet$ \\
$\bullet$ & $\bullet$ & $\bullet$ & $\bullet$ \\
$\bullet \bullet$ & $\bullet$ & $\bullet$ & $\bullet$ \\
$\bullet$ & $\bullet$ & $\bullet$ & $\bullet$ \\
\end{tabular} & 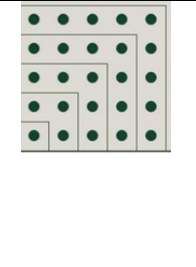 & 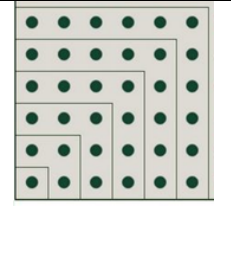 & 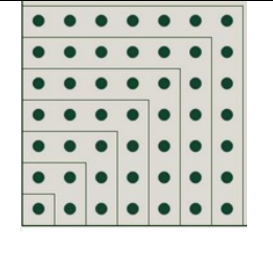 & 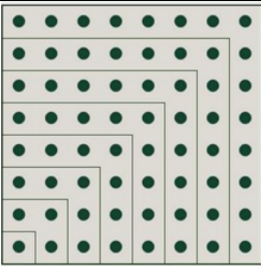 \\
\hline 1 & 4 & 9 & 16 & 25 & 36 & 49 & 64 \\
\hline $1^{2}=1$ & $2^{2}=1+3$ & $\begin{aligned} 3^{2} & =1+3 \\
& +5\end{aligned}$ & $\begin{array}{c}4^{2}= \\
1+3+5+7\end{array}$ & $\begin{array}{c}5^{2}=1+3+5+7 \\
+9\end{array}$ & $\begin{aligned} 6^{2}=1 & +3+5+7+9 \\
& +11\end{aligned}$ & $\begin{array}{c}7^{2}=1+3+5+7+9+1 \\
1+13\end{array}$ & $\begin{array}{c}8^{2}=1+3+5+7+9+ \\
11+13+15\end{array}$ \\
\hline 1 & 2 & 3 & 4 & 5 & 6 & 7 & 8 \\
\hline
\end{tabular}

Figura 5. Descomposición del diagrama e interpretaciones

3. Cada número impar que se suma se puede escribir como el doble de su posición en la secuencia menos 1. Así, el número impar sumado correspondiente a la posición 8 corresponde a:

$$
15=(2 \times 8-1)
$$

4. El número cuadrado $8^{2}$ corresponde a la suma de los ocho primeros números impares:

$$
8^{2}=(2 \times 1-1)+(2 \times 2-1)+(2 \times 3-1)+(2 \times 4-1)+(2 \times 5-1)+(2 \times 6-1)+(2 \times 7-1)+(2 \times 8
$$

5. La secuencia de pasos anteriores permite conjeturar que la suma de los $n$ primeros números impares se puede calcular mediante la expresión:

$$
1+3+\cdots+(2 n-1)=n^{2}(\mathrm{~A})
$$

6. La expresión (A) se ha comprobado que es válida para los ocho primeros números impares. El paso de un caso al siguiente supone añadir el número impar siguiente de puntos, dispuestos en el ángulo superior derecho del cuadrado anterior. Esto supone añadir $n+n+1=2 n+1$ puntos a los $n^{2}$ puntos anteriores, obteniendo un nuevo cuadrado de lado $n+1$ (Figura 6).

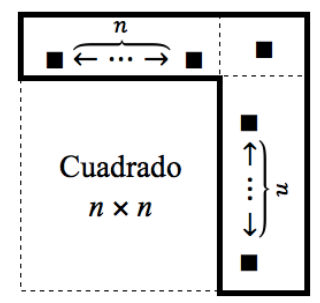

Figura 6. Composición del cuadrado que ocupa el lugar $n+1$ a partir del que ocupa el lugar $n$. 
Por tanto, la suma de los $n+1$ números impares es $(n+1)^{2}$. En efecto, en aplicación del principio de inducción se tiene:

a) $\operatorname{Si} n=1: 1=1^{2}$.

b) Si la expresión A es válida para la suma de los $n$ primeros números impares, entonces es cierta para la suma de los $n+1$ (hipótesis de inducción, HI):

$$
[1+3+\ldots+(2 n-1)]+(2 n+1) \underset{H I}{=} n^{2}+(2 n+1)=(n+1)^{2}
$$

\subsection{Registros de representación semiótica y configuración ontosemiótica}

En la Tabla 1 sintetizamos los diversos registros de representación semiótica así como los tratamientos y conversiones usados en cada una de las prácticas indicadas en el apartado 4.1. El análisis de la práctica matemática que se sigue de estos registros y sus cambios asociados es completado con la configuración de objetos matemáticos implícitos (no ostensivos). Los procesos de interpretación y comprensión requeridos se incluyen en la Sección 4.3 aplicando la noción de función semiótica, mediante la cual se completa la identificación de la trama de conocimientos que se ponen en juego en la resolución de la tarea.

Tabla 1. Análisis conjunto de las prácticas matemáticas

\begin{tabular}{|c|c|c|}
\hline $\begin{array}{l}\text { Registros de representación } \\
\text { semiótica } \\
\text { (tratamientos y conversiones) }\end{array}$ & $\begin{array}{l}\text { Prácticas operativas y } \\
\text { discursivas textualizadas }\end{array}$ & $\begin{array}{c}\text { Objetos no ostensivos } \\
\text { (conceptos, proposiciones, } \\
\text { procedimientos, argumentos) }\end{array}$ \\
\hline $\begin{array}{l}\text { - RR lengua natural, RR figural y } \\
\text { RR numérico. } \\
\text { - Conversión no congruente entre } \\
\text { RR lengua natural y figural. }\end{array}$ & $\begin{array}{l}\text { Enunciado: ¿Cuánto suman los } \\
n \text { primeros números impares? } \\
\text { Resuelve la tarea usando un } \\
\text { razonamiento de tipo visual } \\
\text { apoyado en el diagrama } \\
\text { adjunto. }\end{array}$ & $\begin{array}{l}\text { - Conceptos: números naturales e } \\
\text { impares; orden y adición en } \mathrm{N} \text {; cardinal } \\
\text { de un conjunto; números figurados. } \\
\text { - Argumento: razonamiento visual. }\end{array}$ \\
\hline $\begin{array}{l}\text { - RR lengua natural, RR figural y } \\
\text { RR numérico. } \\
\text { - Tratamiento dentro del RR figural } \\
\text { descomponiendo un diagrama en } \\
\text { ocho sub-diagramas, visualizando } \\
\text { solo la disposición angular de los } \\
\text { impares. } \\
\text { - Correspondencia entre los sub- } \\
\text { diagramas y sus respectivas } \\
\text { posiciones en la secuencia numérica } \\
\text { (conversión congruente). }\end{array}$ & $\begin{array}{l}\text { 1) El diagrama de la Figura } 3 \text { se } \\
\text { debe descomponer en ocho sub- } \\
\text { diagramas (Figura 4) según las } \\
\text { líneas angulares interiores. La } \\
\text { secuencia de las } 8 \text { figuras } \\
\text { establece una correspondencia } \\
\text { entre los primeros } 8 \text { números } \\
\text { impares y el número de orden } \\
\text { de la figura. }\end{array}$ & $\begin{array}{l}\text { - Procedimiento: descomposición de un } \\
\text { diagrama (tratamiento) } \\
\text { - Concepto: número natural como } \\
\text { secuencia de numerales; } \\
\text { función de variable natural que relaciona } \\
\text { la posición de la figura con el impar } \\
\text { "figurado angularmente". }\end{array}$ \\
\hline $\begin{array}{l}\text { - RR lengua natural, RR figural y } \\
\text { RR numérico } \\
\text { - Tratamiento dentro del RR figural } \\
\text { componiendo ocho sub-diagramas a } \\
\text { partir de los diagramas de la Figura } \\
\text { 4, visualizando los "números } \\
\text { cuadrados". } \\
\text { - Conversión del RR figural al RR } \\
\text { numérico en cada sub-diagrama } \\
\text { cuadrangular. }\end{array}$ & $\begin{array}{l}\text { 2) En cada caso se calcula el } \\
\text { número de puntos de dos } \\
\text { formas: como área discreta de } \\
\text { un cuadrado ("números } \\
\text { cuadrados) y como suma de } \\
\text { números impares (Figura 5). }\end{array}$ & $\begin{array}{l}\text { - Concepto: cardinal de un conjunto de } \\
\text { puntos. } \\
\text { - Procedimientos: } \\
\text { composición de la disposición } \\
\text { cuadrangular de los puntos a partir de } \\
\text { las figuras angulares; } \\
\text { paso del registro gráfico al numérico- } \\
\text { aritmético; multiplicación como } \\
\text { producto cartesiano (área discreta de } \\
\text { cada cuadrado). } \\
\text { - Proposiciones: }\end{array}$ \\
\hline
\end{tabular}




\begin{tabular}{|c|c|c|}
\hline & & $\begin{array}{l}1^{2}=1 ; 2^{2}=1+3=; \ldots \\
- \text { Argumentos: comprobación visual y } \\
\text { aritmética }\end{array}$ \\
\hline $\begin{array}{l}\text { - RR lengua natural; RR numérico. } \\
\text { - Tratamiento dentro del RR } \\
\text { numérico. }\end{array}$ & $\begin{array}{l}\text { 3) Todo número impar se puede } \\
\text { escribir como } 2 \times n-1 \text {, donde } n \\
\text { representa su posición en la } \\
\text { secuencia natural. }\end{array}$ & $\begin{array}{l}\text { - Proposiciones: } \\
15=(2 \times 8-1) \\
\text { - Argumento: comprobación mediante } \\
\text { cálculo aritmético }\end{array}$ \\
\hline $\begin{array}{l}\text { - RR lengua natural; RR numérico. } \\
\text { - Tratamiento dentro del RR } \\
\text { numérico. }\end{array}$ & $\begin{array}{l}\text { 4) El número cuadrado } 8^{2} \\
\text { corresponde a la suma de los } 8 \\
\text { primeros números impares: } \\
8^{2}=(2 \times 1-1)+\ldots+(2 \times 8-1)\end{array}$ & $\begin{array}{l}\text { - Procedimiento: cálculo aritmético } \\
\text { (tratamiento dentro del registro } \\
\text { numérico). }\end{array}$ \\
\hline $\begin{array}{l}\text { - RR lengua natural; RR algebraico. } \\
\text { - Tratamiento dentro del RR } \\
\text { numérico. } \\
\text { - Conversión del RR numérico al } \\
\text { RR algebraico. }\end{array}$ & $\begin{array}{l}\text { 5) La secuencia de pasos } \\
\text { anteriores permite conjeturar } \\
\text { que la suma de los } n \text { primeros } \\
\text { números impares se puede } \\
\text { calcular mediante la expresión: } \\
1+3+\ldots+(2 n-1)=n^{2}\end{array}$ & $\begin{array}{l}\text { - Concepto: variable } n \text { que expresa una } \\
\text { posición genérica en la serie de números } \\
\text { naturales. } \\
\text { - Proceso: generalización inductiva. } \\
\text { - Proposición: } \\
1+3+\ldots+(2 n-1)=n^{2} \\
\text { - Argumento: justificación de la } \\
\text { proposición mediante la secuencia de } \\
\text { pasos previos (para un valor fijo de } n \\
\text { aunque indeterminado). }\end{array}$ \\
\hline $\begin{array}{l}\text { - RR lengua natural; RR algebraico; } \\
\text { RR figural (evocado). } \\
\text { - Conversión del RR figural } \\
\text { (evocado) al RR algebraico. } \\
\text { - Tratamiento dentro del RR } \\
\text { algebraico. }\end{array}$ & $\begin{array}{l}\text { 6) La expresión (A) se ha } \\
\text { comprobado que es válida para } \\
\text { los } 8 \text { primeros números } \\
\text { impares... Por el principio de } \\
\text { inducción se demuestra que la } \\
\text { suma de los } n+1 \text { números } \\
\text { impares es }(n+1)^{2} \text {. }\end{array}$ & $\begin{array}{l}\text { - Proposición: la expresión A es válida } \\
\text { para todo } n \text { natural. } \\
\text { - Argumento: verificación por el } \\
\text { principio de inducción matemática. } \\
\text { - Procedimiento: razonamiento de } \\
\text { inducción matemática. }\end{array}$ \\
\hline
\end{tabular}

La conversión y tratamiento de la TRRS pueden ser asimilados sin tensiones por el EOS como procedimientos en las prácticas operativas y discursivas. Así, por ejemplo, la práctica 2, la composición-descomposición de los puntos que representan los números impares (escuadras) y su suma (cuadrado) es un procedimiento que supone un tratamiento figural. Por otro lado, el procedimiento que permite la interpretación numérica de la representación gráfica es una conversión.

\subsection{Identificación de funciones semióticas}

Los tratamientos y conversiones son tipos de prácticas matemáticas y como tales ponen en juego no solo la "manipulación" de objetos ostensivos sino también de no ostensivos. Tales manipulaciones involucran una trama de funciones semióticas cuya identificación permite revelar la complejidad de los procesos de interpretación y actuación requeridos, así como la lógica e intencionalidad de cada acción. Un acto de interpretación o semiosis pone en relación un objeto antecedente con otro consecuente según un cierto convenio o regla de correspondencia. El acto de semiosis puede consistir en dar sentido, uso o finalidad, a una acción, o secuencia de acciones, en el marco de la actividad en desarrollo.

Cada práctica en que se ha dividido el proceso resolutivo del problema se puede analizar en términos de la trama de objetos matemáticos (ostensivos y no ostensivos) y de las funciones semióticas que se establecen entre los mismos. El análisis ontosemiótico va a requerir descomponer las prácticas (primarias) inicialmente enunciadas en otras más 
elementales. A título de ejemplo mostramos a continuación las funciones semióticas implicadas en el enunciado y en las prácticas 1) y 2).

\section{Enunciado}

a. Los términos, 'cuánto', 'suman', 'primeros números impares', refieren a sus respectivos contenidos conceptuales, que vienen definidos en el contexto matemático.

b. 'Razonamiento visual' refiere, en el contexto escolar, a una secuencia de prácticas discursivas y operativas que se deben realizar sobre el diagrama dado las cuales conducirán finalmente a una respuesta a la cuestión planteada.

c. La letra $n$ refiere a un número natural cualquiera, y por tanto, asociado a la noción de variable y, en el contexto, la necesidad de argumentación de la validez por medio de la inducción matemática. Así, la respuesta esperada es una expresión algebraica que sintetice las relaciones y su interpretación en los registros del lenguaje natural, figural y simbólico.

d. 'Diagrama adjunto' refiere a la disposición geométrica cuadrangular de puntos y los 8 primeros números impares de la Figura 3; en estas funciones semióticas ambos funtivos son ostensivos.

e. El texto completo del enunciado es referido como 'enunciado de la tarea', como una consigna que hay que interpretar y que induce a desplegar una actividad matemática específica regulada por los significados convenidos para los distintos términos y expresiones.

\section{Práctica 1}

a. El sujeto interpreta las líneas angulares encajadas de la Figura 3 como la superposición de 8 casos, aplicando un tratamiento visual guiado por las líneas angulares interiores. Tales líneas tienen un significado actuativo u operatorio, es decir, sugieren tácitamente la operación de descomposición ostensiva de la Figura 3 en 8 sub-figuras.

b. La finalidad, el uso o significado operativo de estas acciones es la obtención de la secuencia de los primeros 8 números impares, cada uno representado como un "impar figural angular".

c. El objeto figural impar se pone en correspondencia con la secuencia de números naturales del 1 al 8 , con la finalidad de producir la función $f(n)=2 n-1$, particularizada a los 8 primeros números naturales.

d. Se trata de una conversión del registro figural al numérico que es congruente porque cada unidad del registro de partida se corresponde con una unidad correspondiente en el de llegada.

\section{Práctica 2}

a) Tratamiento figural para pasar de las disposiciones angulares de los impares a los "números cuadrados". Para el primer caso, la unión de un punto junto con tres puntos (antecedente) produce un cuadrado (consecuente). En cierto modo podríamos decir que el cuadrado "significa" el resultado de agregar dos números impares figurales, en el sentido de que es el consecuente de la función semiótica que los relaciona. Este tratamiento se usa, o tiene como fin en esta práctica, para relacionar la suma de impares con los números cuadrados. 
b) Conversión 1: Correspondencia entre cada conjunto de puntos dispuestos en forma cuadrangular y el cardinal correspondiente (número de puntos); donde:

- Antecedente: figural.

- Consecuente: numérico.

- Criterio: convencional (el número de puntos se expresa con los numerales correspondientes en el sistema posicional decimal).

Es pues una conversión congruente: cada unidad significante del registro de partida (figuras cuadrangulares de puntos) se corresponde con la potencia de orden 2 del lado correspondiente. La disposición tabular de figuras y números establece un mismo orden de aprehensión de las unidades.

c) ¿Qué significado, uso o intencionalidad tiene la conversión 1? Es un paso auxiliar previo al tratamiento que produce la expresión numérica de los "números cuadrados".

d) Conversión 2: registro gráfico (disposición angular de puntos) al numérico (suma de impares, $1+3 ; 1+3+5 ; \ldots)$. La disposición cuadrangular se debe interpretar como unión de las disposiciones angulares que la componen, lo que da como resultado la suma de los impares correspondientes. Se pretende poner en relación la suma de impares con los números cuadrados previamente identificados. Es una conversión congruente porque hay correspondencia entre las unidades significantes de cada registro.

e) Tratamiento numérico 1: Expresión de 1, 4, 9, ., como $1^{2}, 2^{2}, 3^{2}, \ldots$

f) Tratamiento numérico 2: Producción de la equivalencia de las expresiones, $1^{2}=1$, $2^{2}=1+3 ; 3^{2}=1+3+5, \ldots$

Podemos decir que, de este modo, se identifica en cada práctica una "configuración ontosemiótica elemental", constituyendo la secuencia de tales configuraciones una "trayectoria ontosemiótica" del proceso resolutivo. El análisis de los distintos tipos de RRS usados (tratamientos y conversiones; congruentes o no congruentes), se realiza de manera articulada y conjunta con los objetos no ostensivos imbricados, lo cual permite reconocer de manera explícita la sinergia entre la semiosis y la noesis. En el EOS, la noesis se puede describir como un proceso de interpretación y "manipulación" de los objetos no ostensivos que participan en las prácticas matemáticas, en conjunción dialéctica con los ostensivos que necesariamente les acompañan.

\section{Configuración cognitiva de una solución al problema visual - algebraico}

En este apartado analizamos la respuesta dada por un estudiante al problema planteado en la sección anterior, aplicando las herramientas configuración ontosemiótica y registros de representación semiótica. La solución está reflejada en la Figura 7 y fue seleccionada entre las respuestas dadas por futuros docentes en un Máster en Profesorado de Matemáticas en Educación Secundaria. Este problema (Figura 3) es usado para promover la reflexión de los estudiantes sobre los objetos y procesos implicados en la actividad matemática. En nuestro caso, el análisis que realizamos trata de mostrar la potencial utilidad del análisis epistémico realizado en la Sección 4 para interpretar y comprender la configuración cognitiva movilizada por el estudiante e identificar algunas limitaciones metodológicas.

1) Observamos el diagrama que se adjunta para extraer un patrón que nos sirva para ver la relación existente entre números impares y números cuadrados.

2) Observando el diagrama obtenemos los siguientes resultados: 


\begin{tabular}{|l|}
$\begin{array}{l}1=1^{2} \\
1+3=4=2^{2} \\
1+3+5=9=3^{2} \\
\cdots \\
1+3+5+\cdots(2 n-1)=n^{2}\end{array}$ \\
3) Por lo tanto vemos que la suma de los n primeros números impares es, \\
efectivamente $n^{2}$
\end{tabular}

Figura 7. Solución de un estudiante

En la Tabla 2 indicamos los objetos no ostensivos puestos en juego en las prácticas matemáticas manifestadas por el estudiante, así como los registros de representación semiótica (RRS), clasificados según el registro: lenguaje natural (RRL), numérico (RRN), algebraico (RRA) o figural (RRF).

En términos de la teoría de los registros de representación semiótica (TRRS), el estudiante comienza realizando de manera implícita o mental un tratamiento dentro del registro de representación figural (RRF). Este tratamiento le permite al sujeto establecer relaciones diagramáticas, y luego pasar del RRF y de la lengua natural (enunciado de la tarea) al registro de representación numérico ( $R R N$ ) (suma de números impares consecutivos y su relación con los números cuadrados). En el RRN se realizan determinados tratamientos, para finalmente pasar al registro algebraico: $1+3+5+\ldots+(2 n-1)=n^{2}$. Pero como se muestra en la secuencia de prácticas 1) a 3), el RRF no se manifiesta explícitamente como recurso de soporte para hallar la solución; en este caso, el tratamiento de los diagramas figurales resulta costoso y poco operativo.

Tabla 2. Análisis conjunto de las prácticas matemáticas de un estudiante

\begin{tabular}{|c|c|c|}
\hline $\begin{array}{l}\text { Registros de representación } \\
\text { semiótica } \\
\text { (Tratamientos y conversiones) }\end{array}$ & $\begin{array}{c}\text { Prácticas operativas y } \\
\text { discursivas textualizadas }\end{array}$ & $\begin{array}{c}\text { Objetos no ostensivos } \\
\text { (Conceptos, proposiciones, } \\
\text { procedimientos, argumentos) }\end{array}$ \\
\hline $\begin{array}{l}\text { - RR figural (presente en el } \\
\text { enunciado). } \\
\text { - RRL }\end{array}$ & $\begin{array}{l}\text { 1) Observamos el diagrama que } \\
\text { se adjunta para extraer un patrón } \\
\text { que nos sirva para ver la relación } \\
\text { existente entre números impares } \\
\text { y números cuadrados. }\end{array}$ & $\begin{array}{l}\text { - Conceptos: diagrama; patrón; } \\
\text { números impares, números } \\
\text { cuadrados; } \\
\text { - Proposición: relación entre } \\
\text { números impares y números } \\
\text { cuadrados. }\end{array}$ \\
\hline $\begin{array}{l}\text { - RRF; RRL; RRN; RRA } \\
\text { - Tratamiento implícito dentro del } \\
\text { RR figural (interpretación y } \\
\text { descomposición del diagrama de la } \\
\text { Figura 3. Se visualizan los números } \\
\text { impares y cuadrados). } \\
\text { - Conversión del RRF al RRN de } \\
\text { cada sub-diagrama formado por las } \\
\text { líneas angulares. } \\
\text { - Tratamiento dentro del RRN (se } \\
\text { relacionan la suma de números } \\
\text { impares consecutivos con los } \\
\text { números cuadrados). } \\
\text { - Conversión del RRN al RRA }\end{array}$ & $\begin{array}{l}\text { 2) Observando el diagrama } \\
\text { obtenemos los siguientes } \\
\text { resultados: } \\
\qquad \begin{array}{l}1=1^{2} \\
1+3=4=2^{2} \\
1+3+5=9=3^{2} \\
\ldots \\
1+3+5+\ldots+(2 n-1)=n^{2}\end{array}\end{array}$ & $\begin{array}{l}\text { - Conceptos: diagrama; número } \\
\text { impar; números cuadrados; igualdad } \\
\text { aritmética (resultado de operaciones); } \\
\text { variable } n \text { que expresa una posición } \\
\text { genérica en la serie de números } \\
\text { naturales. } \\
\text { - Procedimiento: } \\
1^{\circ} \text { Descomposición (implícita) del } \\
\text { diagrama de la Figura } 3 \text { siguiendo las } \\
\text { líneas angulares interiores (tratamiento } \\
\text { RRF). } \\
2^{\circ} \text { Conversión del registro gráfico al } \\
\text { numérico - aritmético [se asocian los } \\
\text { puntos como suma de números } \\
\text { impares y con el área discreta del }\end{array}$ \\
\hline
\end{tabular}




\begin{tabular}{|l|l|l|}
\hline $\begin{array}{l}\text { (generalización para un número } \\
\text { cualquiera } n) .\end{array}$ & $\begin{array}{l}\left.\text { cuadrado y, esto es p. e.: } 1+3=4=2^{2}\right] \\
3^{\circ} \text { Cálculo aritmético (tratamiento) } \\
- \text { Proposición } 1+3+\ldots+(2 n-1)=n^{2} \\
- \text { Argumento: justificación de la } \\
\text { proposición mediante la secuencia de } \\
\text { pasos previos (asignando un valor fijo } \\
\text { de } n \text { aunque indeterminado). } \\
- \text { Proceso: generalización inductiva }\end{array}$ \\
\hline - RRL & & $\begin{array}{l}- \text { Proposición: } \text { La expresión dada en } \\
\text { la práctica anterior es válida para todo } \\
n \text { natural. }\end{array}$ \\
\hline
\end{tabular}

El estudiante encuentra la solución finalmente mediante un procedimiento aritméticoalgebraico de cálculo basándose en un proceso de generalización inductiva, que acepta como suficiente. La demostración por inducción matemática de la fórmula encontrada para todo número natural, tiene que ser realizada entonces de forma magistral por el docente.

En la práctica 2), a partir de tratamientos y conversiones no explicitados, el estudiante construye las sumas consecutivas de los números impares; de esta manera se obtiene una relación a partir de la cual la suma de los números impares se relaciona con los números cuadrados correspondientes. El estudiante realiza una serie de cálculos aritméticos logrando establecer una proposición que considera solución de la tarea. No obstante, no es consciente de que con el proceso inductivo que sigue lo que obtiene es una conjetura cuya validez, para cualquier número natural, requiere aplicar el principio de inducción matemática. Dado que se pide una justificación visual sería necesario finalmente construir un diagrama similar al mostrado en la Figura 6.

\section{Comparación y articulación de la TRRS y el EOS}

En esta sección, profundizamos en la comparación y articulación de los dos enfoques teóricos. Como se ha señalado en la Sección 4.2, las nociones de tratamiento y conversión permiten sintetizar procesos en el marco del EOS. Aún más, dado que los lenguajes forman también parte de la ontología matemática del EOS, concreciones del tipo "tratamiento en RRF" o "conversión RRN" pueden asimismo utilizarse sin tensiones en el marco del EOS. Esta asunción, no solo no genera conflictos desde el punto de vista teórico, sino que es extendida mediante la noción de función semiótica (Sección 4.3).

\subsection{Supuestos ontológicos y semióticos de ambos marcos teóricos}

La TRRS asume de manera implícita una posición que podemos calificar de empirista sobre la naturaleza de los objetos matemáticos. Esto se infiere del postulado de que es necesario el uso de dos o más representaciones para la comprensión del objeto, y de que tal objeto es "el invariante de un conjunto de fenómenos o el invariante de alguna multiplicidad de posibles representaciones". La semiótica asumida enfatiza la faceta representacionista/referencial, aunque también se reconoce una valencia instrumental/operacional de las inscripciones simbólicas: "La escritura de un número representa un número y tiene una significación operatoria ligada a los tratamientos que permiten efectuar las operaciones. Los tratamientos no son los mismos para la escritura decimal y para la escritura fraccionaria" (Duval, 1995, p. 64). 
La ontología EOS es pragmatista - antropológica y la semiótica es básicamente peirceanawittgeinsteiniana. Se asume que las representaciones materiales o externas tienen una valencia representacional, de referir a otro objeto no ostensivo, y también una valencia operacional, esto es, los objetos ostensivos se usan para hacer el trabajo matemático sin que tengan que representar a otro objeto. Además, siguiendo la semiótica peirceana, el antecedente de las funciones semióticas pueden ser objetos no ostensivos.

El objeto matemático es visto por la TRRS como 'objeto de conocimiento', como una entidad cognitiva que reside en la mente del sujeto individual, y se plantea como una cuestión esencial el estudio de las operaciones cognitivas necesarias para la realización de diferentes tipos de tareas matemáticas, sean un cálculo, un razonamiento o el uso de una figura en una demostración geométrica. Cuando un sujeto no es capaz de realizar una determinada conversión o tratamiento desde la TRRS solo se puede decir que carece del conocimiento del objeto matemático correspondiente. Es aquí donde el EOS puede complementar los análisis representacionales de la TRSS.

¿Por qué son necesarias las representaciones semióticas en la actividad matemática? ¿Qué relación existe entre el objeto matemático y sus diversas representaciones? La adopción del postulado antropológico para los objetos no ostensivos (conceptos, proposiciones, procedimientos) permite afrontar esas cuestiones. Los objetos matemáticos son reglas gramaticales de los lenguajes que usamos para describir nuestros mundos, por lo que el uso de tales lenguajes (representaciones semióticas) es imprescindible. No puede haber gramática sin lenguaje. Aún más, las reglas gramaticales no se deben confundir con el enunciado lingüístico de las mismas.

\subsection{Función semiótica versus representación}

La noción de registro de representación semiótica, sus tipos, los tratamientos y conversiones entre registros aportan un recurso analítico que desarrolla y complementa a la categoría del objeto primario 'lenguaje' del EOS, pudiendo ser incorporada sin ningún problema. La TRRS amplía la categoría del lenguaje del EOS distinguiendo distintos tipos y desvelando el papel esencial de las transformaciones que se realizan entre (y dentro) los distintos tipos de lenguaje, ahora considerados como RRS. Dada la naturaleza intradiscursiva de los objetos matemáticos, parece necesario tener en cuenta la trama de objetos que se ponen en juego en las transformaciones realizadas con las representaciones semióticas.

La dualidad ejemplar-tipo (extensivo-intensivo) se aplica a todos los tipos de objetos primarios, incluyendo los elementos lingüísticos. Esto permite interpretar que la relación entre 'representación semiótica' y 'registro de representación semiótica' es del tipo extensivointensivo. Una representación semiótica es un ejemplar particular, un registro es un tipo o clase de representación. Entre los elementos constituyentes de los tipos de representación se debe poder hacer con ellos determinadas transformaciones siguiendo un conjunto de reglas.

La noción de función semiótica amplia la noción de representación (Godino \& Font, 2010). La semiótica pragmatista/antropológica asumida por el EOS propone que los objetos que se ponen en correspondencia en las funciones semióticas (funtivos) no son solamente objetos lingüísticos ostensivos (palabras, símbolos, expresiones, diagramas), sino que los conceptos, proposiciones, procedimientos, argumentos, incluso las situaciones pueden ser también antecedentes de las funciones semióticas. Los funtivos también pueden ser entidades unitarias o sistémicas, particulares o generales, materiales o inmateriales, personales o institucionales. 
En el ejemplo analizado en la Sección 4 hemos mostrado que la noción de función semiótica permite analizar de manera microscópica la secuencia de pasos implicados tanto en los tratamientos como en las conversiones, identificando los objetos antecedentes, consecuentes y reglas de correspondencia que se movilizan en cada caso. De ese modo, se pueden explicar los conflictos potenciales o efectivos que tienen lugar en tales transformaciones semióticas en términos de los conocimientos matemáticos específicos requeridos en cada caso. En las conversiones entre registros se ponen en juego tramas de funciones semióticas en las que los antecedentes y consecuentes corresponden a tipos (registros) de representaciones diferentes (p. e., pasaje de lenguaje natural a simbólico). Del mismo modo, en cada paso de un tratamiento el criterio de la correspondencia semiótica puede ser conflictivo para el estudiante de matemáticas (Rojas, 2015).

Cada RRS usado para representar y operar con un objeto matemático proporciona un significado específico para dicho objeto. La comprensión del objeto en su integridad supone o requiere la articulación de los diferentes significados parciales (o sentidos), lo cual no se logra de manera espontánea. El uso de los lenguajes natural, numérico (decimal, fraccionario), algebraico, diagramas, figuras geométricas, gráficos cartesianos, tablas, son RRS diferentes y cada uno plantea cuestiones específicas de aprendizaje. El conocimiento de las reglas de correspondencia entre dos registros diferentes no es suficiente para que puedan ser movilizados y utilizados de manera pertinente.

\section{A modo de conclusión}

Como resultado de los análisis cognitivos hechos con la TRRS se llega a conclusiones del tipo, "hay deficiencias en el conocimiento del sujeto puesto que no realiza la conversión pasaje o tratamiento-". Se detectan las contradicciones en la actividad cognitiva del sujeto y se determina si la tarea se resolvió o no con éxito. Con las herramientas del EOS es posible proporcionar una explicación, desde el punto de vista epistémico, sobre cuáles son las carencias del conocimiento del sujeto a propósito de un problema determinado, al identificar y describir de manera pormenorizada los objetos matemáticos y procesos involucrados en las prácticas matemáticas operativas, discursivas y normativas.

La importancia crucial que se da a la conversión de representaciones (tanto en la enseñanza como en el aprendizaje) desde el punto de vista cognitivo es consecuencia de entender la "comprensión" básicamente en términos de procesos mentales y de asumir un postulado empirista sobre las abstracciones matemáticas. En el EOS se reconoce el papel importante que juegan las diferentes representaciones ostensivas y las traducciones entre ellas en la comprensión matemática (Font \& Peraire 2001), pero se adopta una posición antropológica - pragmatista sobre esta cuestión (Font, Godino, \& Contreras, 2008). Para el EOS, la comprensión alcanzada por un sujeto en un momento dado difícilmente será total o nula, sino que será parcial y progresiva. Se considera que un alumno ha comprendido un determinado contenido cuando lo usa de manera competente en diversas prácticas. Se entiende pues, la comprensión, básicamente, como una capacidad que tiene el alumno y no tanto como un proceso mental que se produce en su mente cuando articula representaciones. La capacidad se traduce en prácticas que son evaluables públicamente ${ }^{1}$, mientras que el proceso mental es una experiencia privada de la persona.

1 Desde el punto de vista del diseño, se pueden definir variables operativas y sus aspectos asociados (indicadores y escalas, instrumentos y procedimientos de medición, así como las formas de expresión final para el análisis). 
Desde un punto de vista ontosemiótico, el problema no es si hay que introducir una sola representación de un objeto o más de una, ni qué traducciones o relaciones entre representaciones hay que tener en cuenta. El problema está realmente en determinar si las representaciones introducidas facilitan, o no, la realización de las prácticas que interesan que formen parte del significado global del alumno, en saber si aumentan o disminuyen la complejidad semiótica y también en saber si hacen emerger o no conflictos semióticos innecesarios.

Así, por ejemplo, en el análisis de la tarea se ha observado que los RRS utilizados por los estudiantes no han generado las condiciones para hacer emerger la necesidad de una demostración formal de la fórmula de la suma de los $n$ primeros números impares. La demostración basada en la RRF (Figura 6) supone una estrategia docente para mejorar la idoneidad didáctica (Godino, Bencomo, Font, \& Wilhelmi, 2006) ya que: a) supone un control mayor sobre la validez de la inducción empírica (idoneidad epistémica), b) se adecúa al significado personal adquirido por el estudiante (idoneidad cognitiva), c) parte de las interacciones previas docente- estudiante y de la resolución de conflictos aparecidos (idoneidad interaccional) y, finalmente, d) contextualiza la propiedad de la suma de los $n$ primeros números impares dentro de un técnica general de demostración (idoneidad ecológica).

\section{Reconocimiento}

Trabajo realizado en el marco de los proyectos de investigación EDU2012-31869 y EDU2013- 41141-P, Ministerio de Economía y Competitividad (MINECO).

\section{Referencias}

Bikner-Ahsbahs, A. \& Prediger, S. (Eds.). (2014). Networking of theories as a research practice in Mathematics Education, Advances in Mathematics Education. Dordrecht, The Netherlands: Springer.

Dickson, L., Brown, M. \& Gibson, O. (1991). El aprendizaje de las matemáticas. Barcelona, España: Labor.

Duval, R. (1995). Sémiosis et pensée: registres sémiotiques et apprentissages intellectuels. Berne, Switzerland: Peter Lang.

Duval, R. (2006). A cognitive analysis of problems of comprehension in a learning of mathematics. Educational Studies in Mathematics, 61(1-2), 103-131.

Eco, U. (1976). Tratado de semiótica general. Barcelona: Lumen.

Font, V., Godino, J. D. \& Contreras, A. (2008). From representation to onto-semiotic configurations in analysing mathematics teaching and learning processes. En, L. Radford, G. Schubring y F. Seeger (Eds.), Semiotics in mathematics education: epistemology, history, classroom, and culture (pp. 157-173). Rotterdam: Sense Publishers.

Font, V., Godino, J. D. \& Gallardo, J. (2013). The emergence of objects from mathematical practices. Educational Studies in Mathematics, 82, 97-124.

Font, V. \& Peraire, R. (2001). Objetos, prácticas y ostensivos asociados. El caso de la cisoide. Educación Matemática, 13(2), 55-67.

Fontes, S., García, C., Quintanilla, L., Rodríguez, R., Rubio \& P. Sarriá, E. (2010). Fundamentos de investigación en psicología. Madrid: UNED. 
Godino, J. D. (2012). Origen y aportaciones de la perspectiva ontosemiótica de investigación en Didáctica de la Matemática. En A. Estepa, A. Contreras, J. Deulofeu, M. C. Penalva, F. J. García \& L. Ordóñez (Eds.), Investigación en Educación Matemática XVI (pp. 49 68). Jaén: SEIEM.

Godino, J.D., Batanero, C. \& Font, V. (2007). The onto-semiotic approach to research in mathematics education. ZDM-The International Journal on Mathematics Education, $39(1), 127-135$.

Godino, J. D., Bencomo, D., Font, V. \& Wilhelmi, M. R. (2006). Análisis y valoración de la idoneidad didáctica de procesos de estudio de matemáticas. Paradigma, 27(2), 221-252.

Godino, J. D. \& Font, V. (2010). The theory of representations as viewed from the ontosemiotic approach to mathematics education. Mediterranean Journal for Research in Mathematics Education, 9(1), 189-210.

Godino, J. D., Font, V., Wilhelmi, M. \& Lurduy, O. (2011). Why is the learning of elementary arithmetic concepts difficult? Semiotic tools for understanding the nature of mathematical objects. Educational Studies in Mathematics, 77(2), 247-265.

Hjelmslev, L. (1943). Prolegomena to a theory of language. Madison, WI: University of Wisconsin Press.

Montiel, M., Wilhelmi, M. R., Vidakovic, D. \& Elstak, I. (2009). Using the onto-semiotic approach to identify and analyze mathematical meaning when transiting between different coordinate systems in a multivariate context. Educational Studies in Mathematics, 72, 139-160.

Ortega, T. \& Pecharomán, C. (2015). Aprendizaje de conceptos geométricos a través de visualizaciones. Avances de Investigación en Educación Matemática, 7, 95-117.

Peirce, C. S. (1978). The collected papers of Charles Sanders Peirce. Cambridge, MA: The Belknap Press of Harvard University.

Pino-Fan, L., Guzmán, I., Duval, R. \& Font, V. (2015). The theory of registers of semiotic representation and the onto-semiotic approach to mathematical cognition and instruction: linking looks for the study of mathematical understanding. En Beswick, K., Muir, T. \& Wells, J. (Eds.), Proceedings of the $39^{\text {th }}$ Conference of the International Group for the Psychology of Mathematics Education (Vol. 4, pp. 33-40). Hobart, Australia: PME Group.

Prediger, S., Bikner-Ahsbahs, A. \& Arzarello, F. (2008). Networking strategies and methods for connection theoretical approaches: first steps towards a conceptual framework. ZDMThe International Journal on Mathematics Education, 40(2), 165-178.

Rojas, P. (2015). Objetos matemáticos, representaciones semióticas y sentidos. Enseñanza de las Ciencias, 33(1), 151-165. 


\title{
Analysing mathematical activity through two theoretical tools: registers of semiotic representation and onto-semiotic configurations
}

\author{
Juan D. Godino, Universidad de Granada (España) \\ Miguel R. Wilhelmi, Universidad Pública de Navarra (España) \\ Teresa F. Blanco, Universidad de Santiago de Compostela (España) \\ Ángel Contreras, Universidad de Jaén (España) \\ Belén Giacomone, Universidad de Granada (España)
}

Specific theoretical tools are necessary to analyse the school mathematical tasks, and the several ways of solving them to understand the potential difficulties and conflicts of learning. In this article, we focus on the epistemic and cognitive analysis of a mathematical task by jointly applying research tools from two different theoretical frameworks: the theory of registers of semiotic representation (TRSR) and the onto-semiotic approach to mathematical cognition and instruction (OSA). The aim of this research is to study the comparison and articulation possibilities of these two theoretical frameworks by using specific tools of each of them, and, at the same time, to establish the potential of each framework and identify their methodological limitations.

Using a task that requires the formulation and proof of a conjecture, and using figural and algebraic representations, we, first of all, provide a possible expert solution, highlighting the necessary mathematical operative as well as discursive actions system. We then, perform an epistemic analysis using each of these theoretical framework to identify the web of knowledge that are put into play in solving the task. This analysis allows us to show the various registers of semiotic representation, their treatments and conversions used in each of the practices, which is completed with the configuration of the mathematical objects, which are mobilized in such practices. In addition, we analyse the interpretation and understanding processes required, through the semiotic function notion.

Additionally, we select an answer to the previously analysed same task, provided by a student -a prospective secondary education mathematics teacher; in this way we continue performing a cognitive analysis of this solution by applying the theoretical tools of each theoretical framework.

With the OSA tools it is possible to provide an explanation, from the epistemic point of view, of the student's knowledge gaps about a particular problem, and to identify and describe in detail the mathematical objects and processes involved in the operative and discursive mathematics practices.

The results reveal some complementarities that allowed us to show the usefulness of the epistemic analysis carried out, identifying effective or potential difficulties and knowledge taking place in the learning processes, and providing teaching strategies to improve the didactical suitability. This article concludes by showing the potential synergy between these tools and the possibility to progress toward the articulation of the corresponding theoretical frameworks. 